TECHNICAL PROGRESS REPORT NO.5

\title{
DEVELOPMENT OF THE INSTRUMENTATION \\ AND MODELING FOR HEAT TRANSFER \\ CHARACTERISTICS IN CFBC
}

TO

U.S. DEPARTMENT OF ENERGY

FEDERAL ENERGY TECHNOLOGY CENTER

P.O. BOX 10940, MS 921-118

PITTSBURGH, PA 15236-0940

FOR

PROJECT NO.: DE-FG22-95MT95013

BY

DR. SEONG W. LEE, PRINCIPAL INVESTIGATOR

MORGAN STATE UNIVERSITY

SCHOOL OF ENGINEERING

BALTIMORE, MD 21239

(phone) 410-319-3137

MAY 1998 
This technical report summarizes the research conducted and progress achieved during the period from October 1, 1997 to March 30, 1998.

Numerical modeling and simulation on the gas velocity and pressure were continued to predict the flow patterns in the CFB. The renormalization group $\mathrm{k}-\varepsilon$ turbulence model was employed to improve the predictions of the near the wall flow, wall heat/mass transfer, and wake/vortex shedding behavior. This model could provide more accurate prediction of swirl than in standard k-E model.

The air velocity profile was predicted in 2 -D velocity vector of the slide plate. The aeration (secondary) air flow rate reduced air velocity and caused a strong gas mixing processes.

The air flowed from the high pressure region into the low pressure region. The aeration air injection affected on its neighborhood pressure profile. Two eddies are formed at level $\mathrm{k}=15$ which is symmetrical with the aeration air inlet center line. It is very interesting to note that the air stream flows from the lower pressure region into the high pressure region.

Experimental measurements will be conducted to understand heat transfer characteristics in the CFB system. In addition, numerical modeling and simulation will be continued to predict the flow patterns, velocity, pressure, temperature, and heat flux with the heat transfer effect in the CFB system. 
TABLE OF CONTENT

PAGE

ABSTRACT

ii

SECTION

1. NUMERICAL SIMULATION FOR THE CFB COLD FLOW MODEL 1

1.1 Basic Physical Modeling 1

1.2 Test Conditions 2

2. RESULTS AND DISCUSSION 4

2.1 The Effect of Air Velocity 4

2.2 The Effect of Air Pressure 5

3. CONCLUSIONS 13

REFERENCES 14 


\section{SECTION 1}

\section{NUMERICAL SIMULATION FOR THE CFB COLD FLOW MODEL}

\subsection{Basic Physical Modeling}

The computational cells and boundaries for the calculation domain are introduced in the previous report [1]. Since the flow with a larger Reynolds number has a strong turbulence flow with unisotropic flow behaviors, the standard k-c turbulence model was not significant for this case. The Renormalization Group (RNG) k- $\varepsilon$ turbulence model [2] can provide more general/fundamental model which yields improved predictions of near the wall flow, wall heat/mass transfer, and wake/vortex shedding behavior [3]. our simulation results showed that the RNG k- $\varepsilon$ model could predict more accurate prediction of swirl than in standard k- $\varepsilon$ models (4).

The RNG k- $\varepsilon$ model uses the Reynolds Averaged Navier Stokes (RANS) equations with the turbulent stresses modeled via the effective viscosity concept $[4,5]$ :

$$
\frac{\partial u_{i}}{\partial t}+\frac{\partial}{\partial x_{j}}\left(u_{i} u_{j}\right)=-\frac{1}{\rho} \frac{\partial p}{\partial x_{i}}+\frac{\partial}{\partial x_{j}}\left[v_{e f f}\left(\frac{\partial u_{i}}{\partial x_{j}}+\frac{\partial u_{j}}{\partial x_{i}}\right)\right]
$$

The effective viscosity required for closure of the momentum equations is computed via the differential form, which is provided by the RNG theory [2]. The RNG theory also provides the transport equations for $\mathrm{k}$ and $\varepsilon$ as,

$$
\frac{\partial k}{\partial t}+u_{i} \frac{\partial k}{\partial x_{i}}=v_{t} S^{2}-\varepsilon+\frac{\partial}{\partial x_{i}} \alpha v_{t} \frac{\partial k}{\partial x_{i}}
$$

and 


$$
\frac{\partial \varepsilon}{\partial t}+u_{i} \frac{\partial \varepsilon}{\partial x_{i}}=C_{1 \varepsilon} \frac{\varepsilon}{k} v_{t} S^{2}-C_{2 \varepsilon} \frac{\varepsilon^{2}}{k}-R+\frac{\partial}{\partial x_{i}} \alpha v_{t} \frac{\partial \varepsilon}{\partial x_{i}}
$$

where a is the inverse Prandtl number for turbulent transport.

The rate-of-strain term $\mathrm{R}$ is given by

$$
R=2 v_{m o l} S_{i j} \frac{\overline{\partial u_{l}^{\prime}}}{\partial x_{i}} \frac{\overline{\partial u_{l}^{\prime}}}{\partial x_{j}}
$$

This term is expressed in the $R N G \mathrm{k}-\varepsilon$ model equations as

$$
R=\frac{C_{\mu} \eta^{3}\left(1-\eta / \eta_{0}\right)}{1+\beta \eta^{3}} \frac{\varepsilon^{2}}{k}
$$

where $\eta=\mathrm{Sk} / \varepsilon, \eta_{0}=4.38$, and $S^{2}=2 S i j, \quad S i j$ is the modulus of the rateof-strain tensor.

\subsection{Test Conditions}

The combustor dimensions, primary air flow condition, and aeration air flow condition are important factors for the test. The lower part of the CFB riser was used as the test section, $135 \mathrm{~cm}$ in height. The aeration (secondary) air inlet at the chamber wall was installed at the height of $15 \mathrm{~cm}$ from the gas distribute plate.

The system was configured in 3-D cylindrical coordinates with uniform mesh grids. The calculation grids was set of orthogonal lines arranges in the cylindrical coordinates, vertical direction (k), radial direction (J), and tangential direction (I) [6]. The lines in the three directions are uniformly spaced.

The test conditions and input boundary conditions are summarized in Table 1.

Table $1 \quad$ Test Conditions for Simulation 
Combustor Diameter

Combustor Height

Aeration Air Nozzle Height

Heat Transfer Probe Diameter

Heat Transfer probe Height

Primary Air Flow Condition:

Air Flow Rate

Air Velocity

Turbulence $(\mathrm{K}-\varepsilon)$

Turbulence-Dissipation

Aeration Air Flow Condition:

Air Flow Rate

Air Velocity

Turbulence $(\mathrm{K}-\varepsilon)$

Turbulence-Dissipation

Gas Density

Gas Viscosity $\mathrm{cm} \quad 11.25$

$\mathrm{cm} \quad 135$

$\mathrm{cm} \quad 15$

$\mathrm{cm} \quad 1.5$

$\mathrm{cm} \quad 35$

$\mathrm{m}^{3} / \mathrm{s} \quad 0.1059$

$\mathrm{m} / \mathrm{s} \quad 10.65$

$\mathrm{m}^{2} / \mathrm{s}^{3} \quad 0.3$

$\mathrm{m}^{2} / \mathrm{s}^{3} \quad 0.3$

$\mathrm{m}^{3} / \mathrm{s} \quad 2.02 \mathrm{e}-3$

$\mathrm{m} / \mathrm{s} \quad 1.03$

$\mathrm{m}^{2} / \mathrm{s}^{2} \quad 0.5$

$\mathrm{m}^{2} / \mathrm{s}^{3} \quad 0.5$

$\mathrm{kg} / \mathrm{m}^{3} \quad 1.2$

N.s $/ \mathrm{m}^{2} 1.72 \mathrm{e}-5$ 
SECTION 2

\section{RESULTS AND DISCUSSION}

\section{$2.1 \quad$ The Effect of Air Velocity}

Figures 1 and 2 show a 2 -D velocity vector in slide plate $\mathrm{I}=4$ and $\mathrm{I}=24$. In the vertical direction, the lower velocity was found at the two ends of the probe, and along the riser wall at the aeration air input side as shown in Figure 1. The flow profiles below the probe were relatively uniform at the center region of the chamber.

When the air flow reached the probe, the uniform flow was spilt to two parts and the velocity of the center region was reduced as shown in Figure 2. The effects of aeration air flow on the flow patterns were on the same side as shown in Figure .2. The lower aeration air flow rate reduced the air flow velocity and caused a strong gas mixing process.

The characteristics of turbulence flow produced some vortex flow in the wall region. This result was clearly shown in Figures 3, 4, and 5 at levels $\mathrm{k}=15,23$, and 30 . A comparison of Figure 3 (untouched probe) with Figures 4 and 5 (touched probe) indicated the vortex flow. This vortex flow increased as the flow touched the probe. This result also proves that the RNG model is very sensitive in accordance with the flow's differential velocities.

At the bottom of the chamber, the velocity was an uniform as the primary air velocity. The center was larger than at the nearer wall region. At the aeration air injection levels, $(\mathrm{k}=6$ and $\mathrm{k}=7)$, the velocity gradient was 
sloped down, since the mixing of both primary flow and aeration air flow reduces the upward flow momentum.

\subsection{The Effect of Air Pressure}

Figure 6 shows the pressure profiles in the same 2-D slides. A high pressure point was found at the bottom of the probe and a lower pressure point was at the top of the probe as shown in Figure 6. The positive numbers of pressure unit indicate the pressure to be larger than the system pressure; the negative numbers indicate the pressure to be smaller than the system pressure. The air flowed from the high pressure region into the lower pressure region.

The aeration air injection also has effects on its neighborhoods as shown in Figure 7 for level $k=2$ and Figure 3 for level $k=15$. A comparison of level $\mathrm{k}=2$ with level $\mathrm{k}=15$ shows a back flow in this region and it also forms two eddies at level $k=15$. The two eddies are symmetrical with the aeration air inlet center line. It is also evidence for the back flow formed in the region where the high pressure region located different slides.

Figure 4 shows the flow profiles at the probe bottom, level k=23. The diameter of the eddies increased and the pressure profile was evened around the center probe. The back flow cross the probe and the eddies continued increasing its size. it is very interesting to note that the air stream flows from the lower pressure region into the high pressure region. This phenomenon may indicate that the back flow had strong momentum to push the flow into a higher pressure region from lower pressure region. 


\section{SECTION 3}

\section{CONCLUSIONS}

Numerical modeling and simulation on the gas velocity and pressure were continued to predict the flow patterns in the CFB. The Renormalization Group (RNG) k- $\varepsilon$ turbulence model was employed to improve the predictions of the near the wall flow, wall heat/mass transfer, and wake/vortex shedding behavior.

The air velocity profile was predicted in 2-D velocity vector of the slide plate. The aeration (secondary) air flow rate reduced the air velocity and caused a strong gas mixing processes. The characteristics of turbulence flow produced some vortex flow in the wall region.

The pressure profiles were showed in 2-D slide plate. The air flowed from the high pressure region into the low pressure region. The aeration air injection affected on its neighborhood pressure profile. A high pressure point was found at the bottom of the probe.

Two eddies are formed at level $\mathrm{k}=15$. These two eddies are symmetrical with the aeration air inlet center line. It is very interesting to note that the air stream flows from the lower pressure region into the high pressure region. The diameter of the eddies increased and the pressure profile was evened around the center probe.

Numerical modeling and simulation will be continued to predict the flow patterns, velocity, pressure, temperature with the heat transfer effect in the CFB system. 


\section{REFERENCES}

[1] Lee, S.W., Technical Progress Report, No.4, U.S. DOE, Federal Energy Technology Center(FETC), October 1997.

[2] Fluent User's Guide, Vol.1, Chapter 6, pp.47-50, 1995.

[3] Boemer, A., et. al, Eulerian Computation of Fluidized Bed Hydrodynamics: A Comparision of Physical Models, Proc. of $13^{\text {th }}$ Int'l Conference on FBC, pp 775-788, 1995

[4] Launder, B.E. and D.B. Spalding, Mathematical Models of Turbulence, Academic Press, London, 1972.

[5] Hoffman, K.A. and S.T. Chiang, Computational Fluid Dynamics 3rd Ed., vol.1, Chapt.9, Vol.2, Chapters $11 \& 13$, Engineering Education System, KS. 1995. 


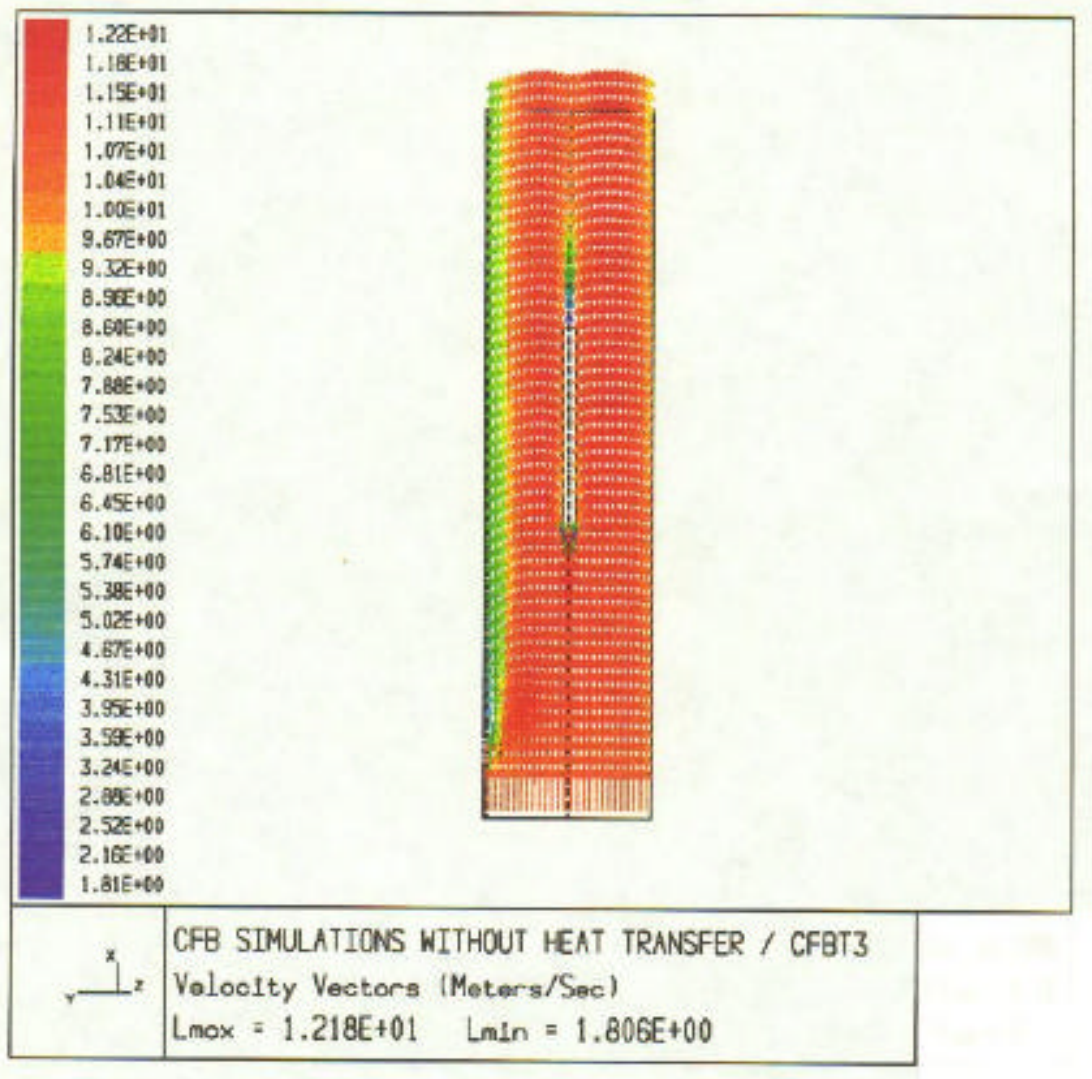

Figure 1 Velocity Vector along the CFB Riser 


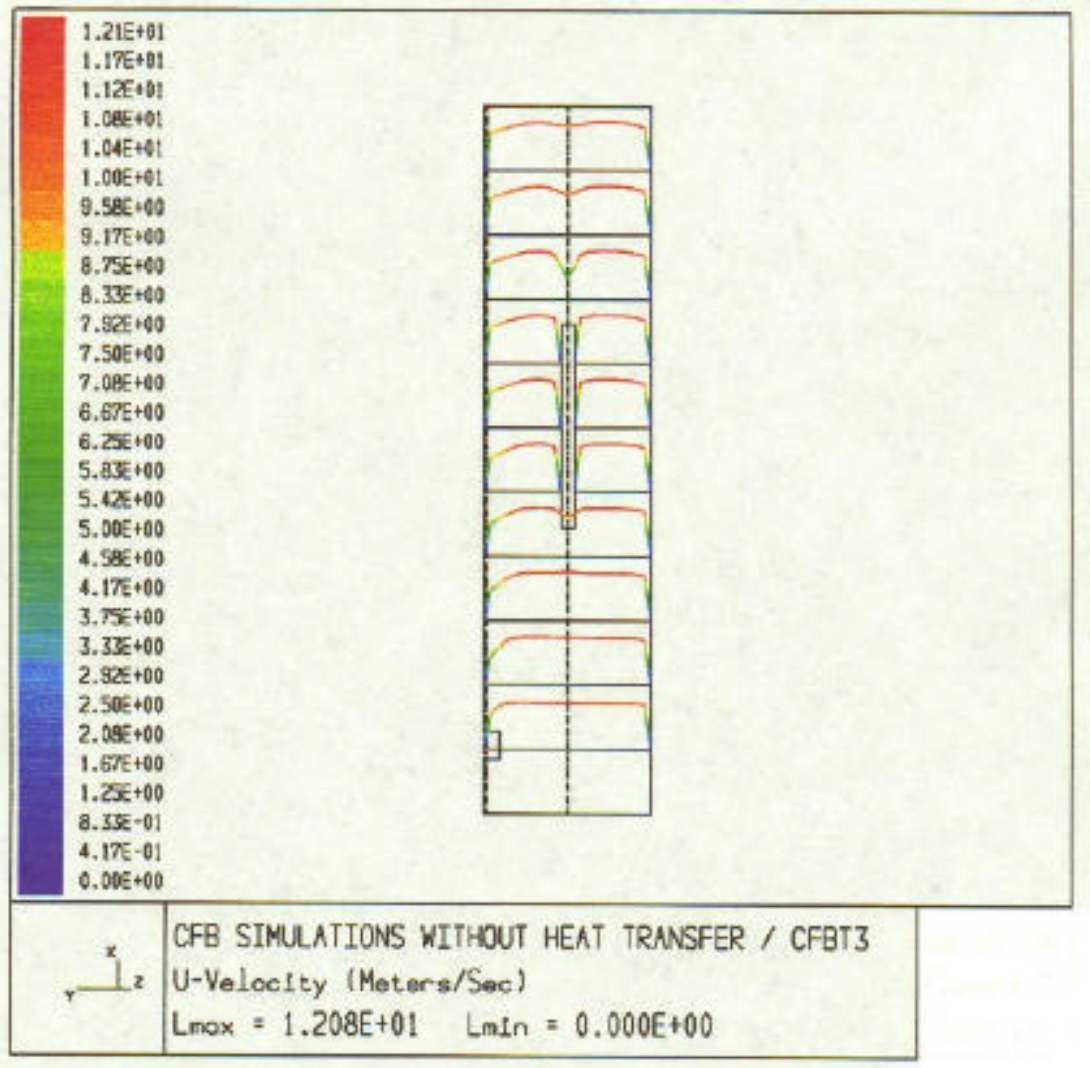

Figure 2 Velocity Profiles in the Vertical Direction of CFB Riser 


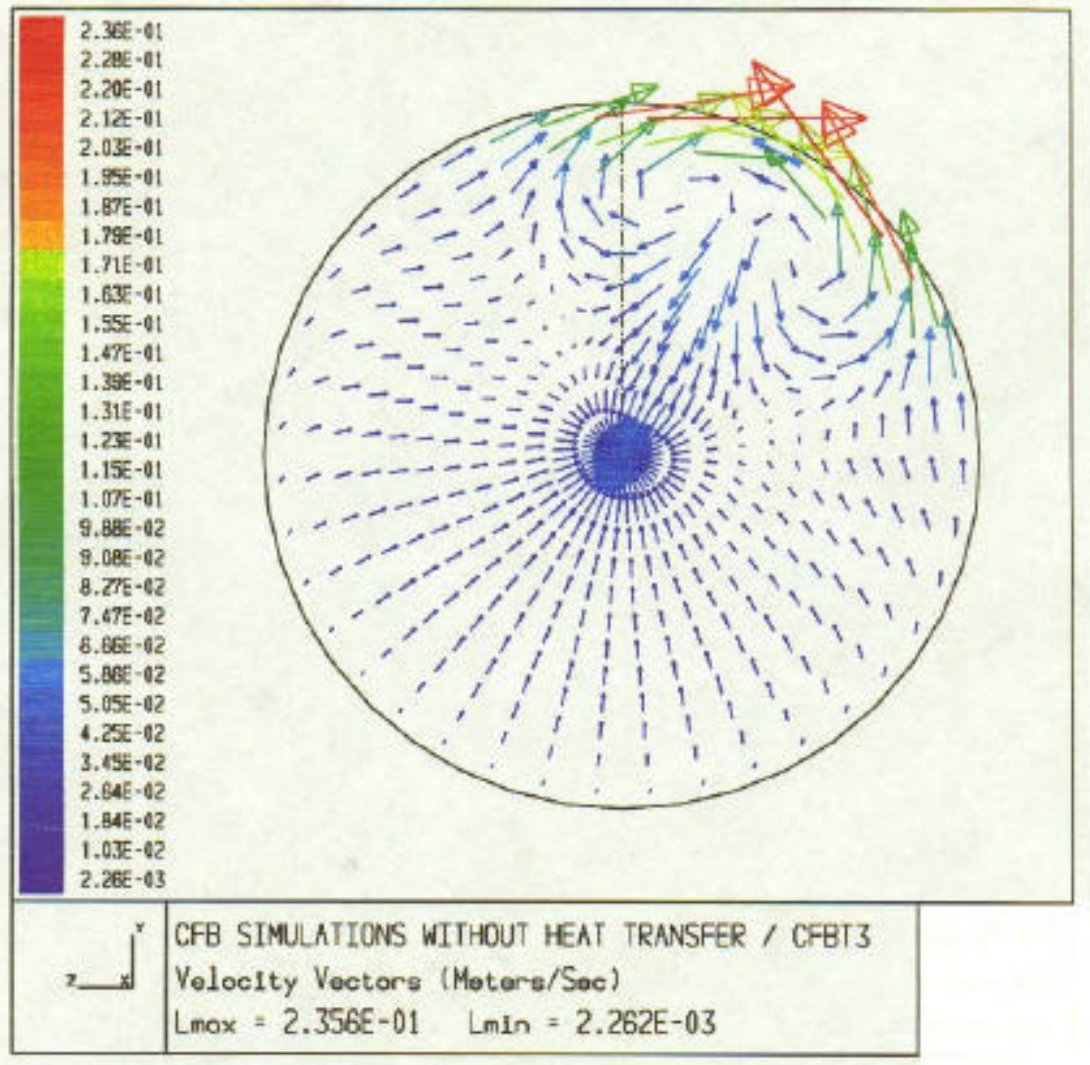

Figure 3 Velocity Vector at Level, k=15 


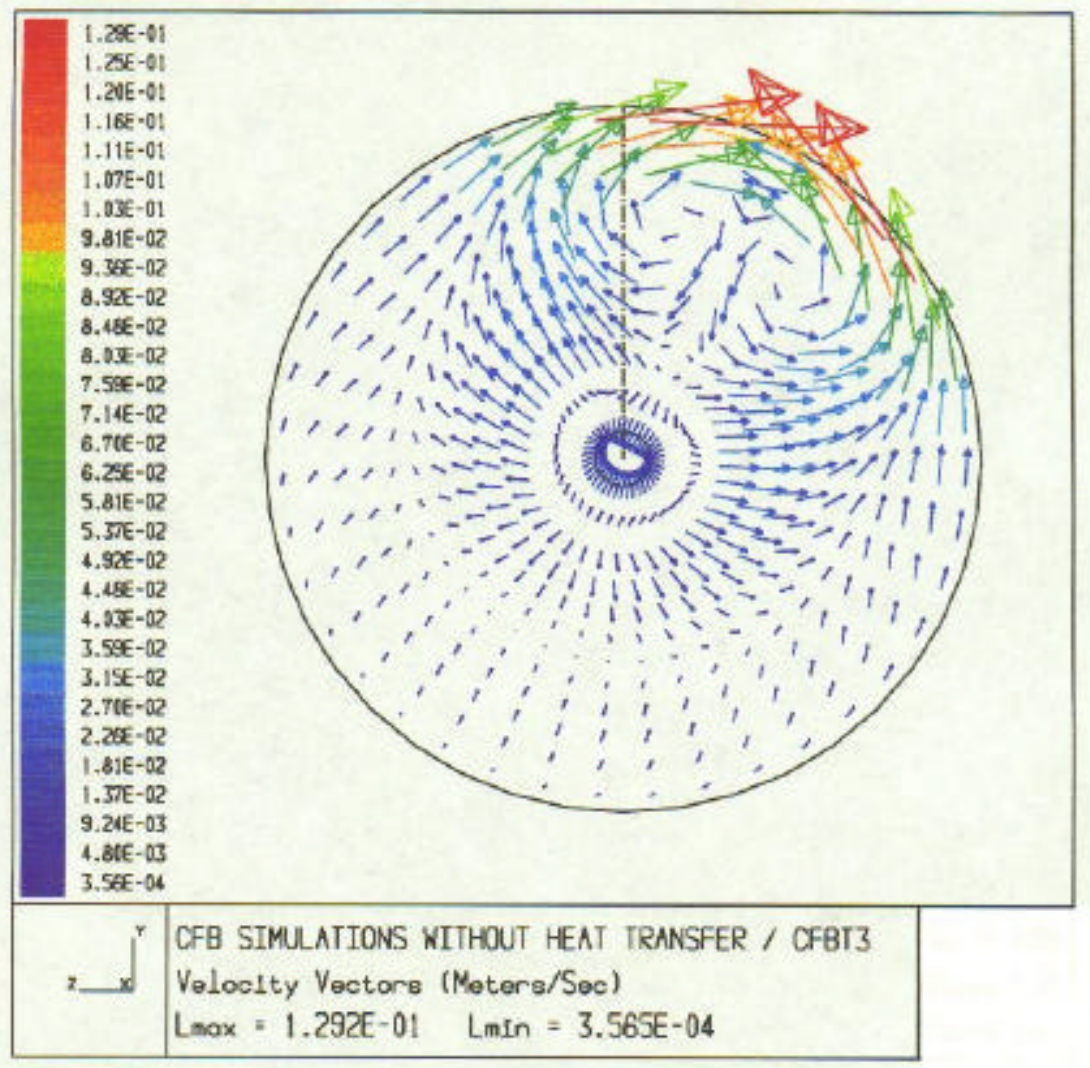

Figure 4 Velocity Vector at Level, k=23 


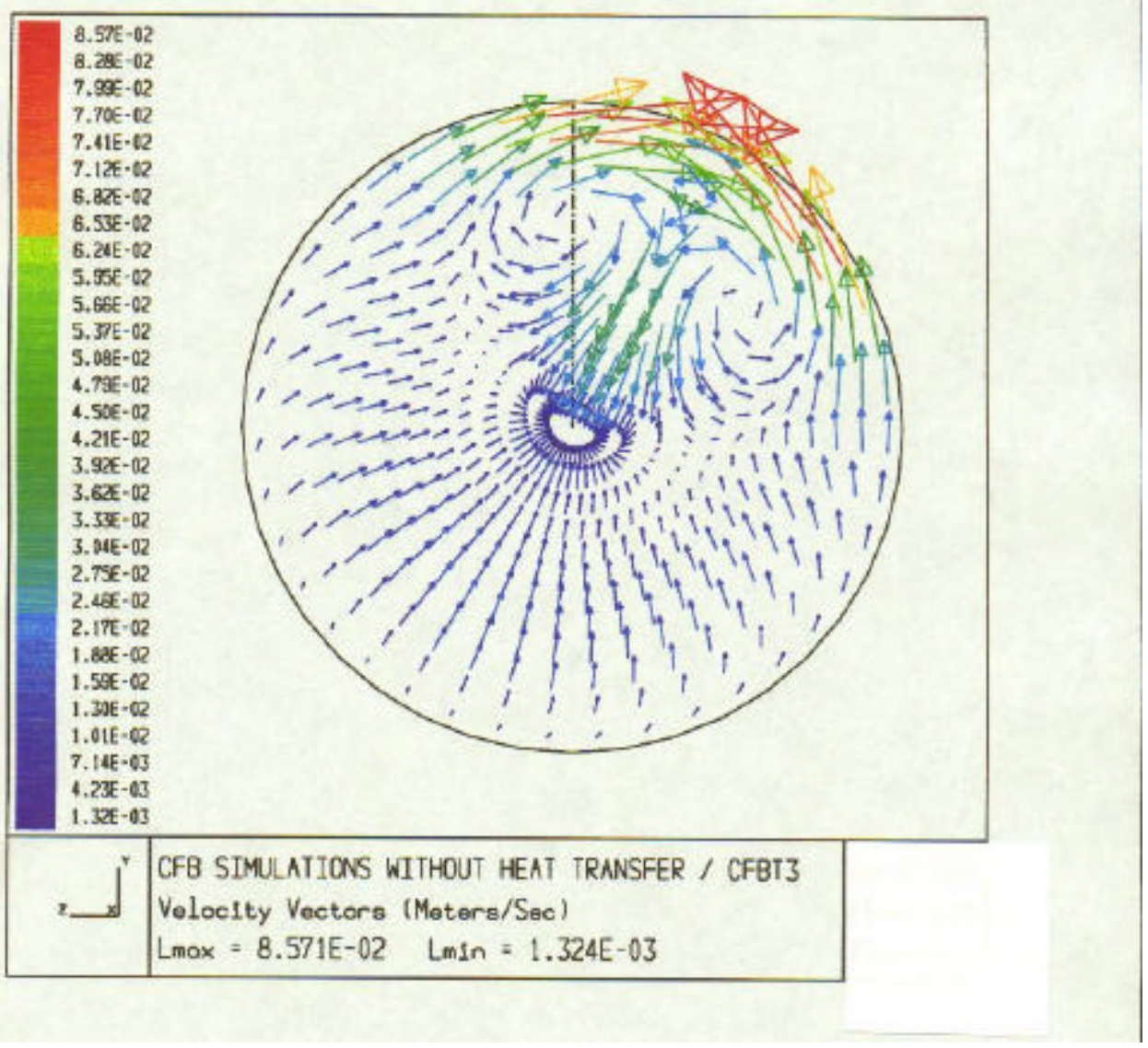

Figure 5 Velocity Vector at Level, k=30 


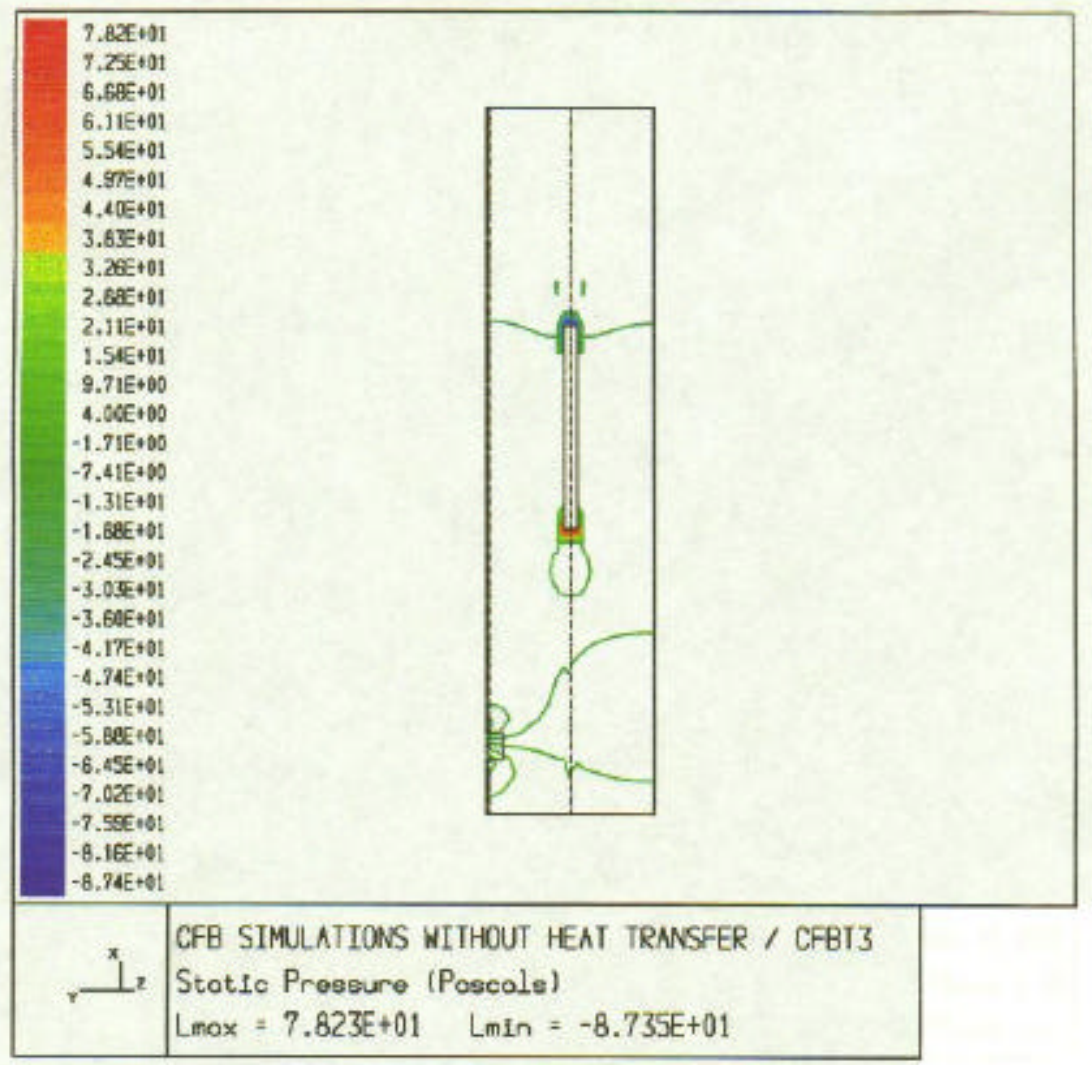

Figure 6 Pressure Profiles along the CFB Riser 


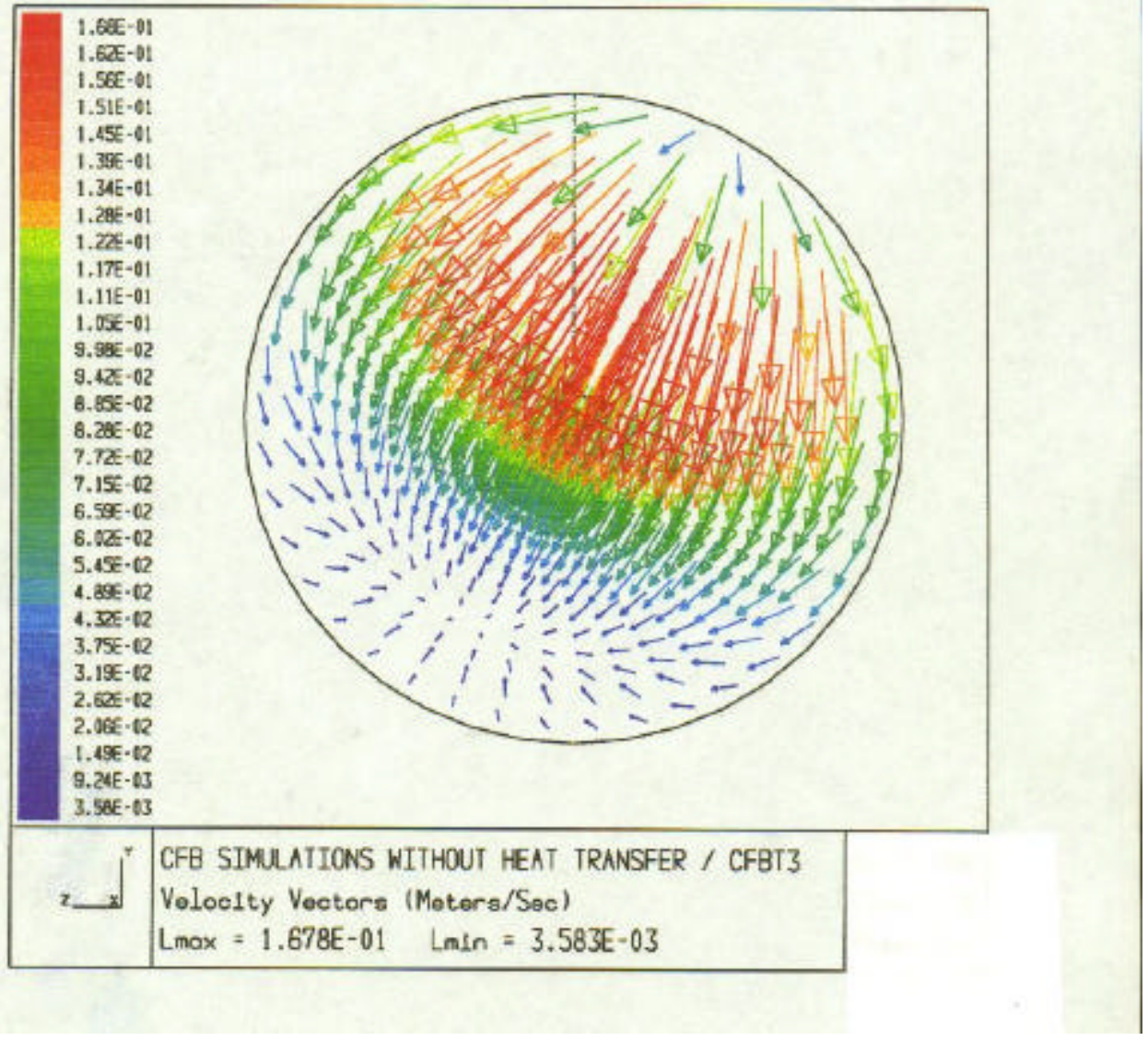

Figure 7 Velocity Vector at Level, k=2 